\title{
Vaccination antigrippale et autonomie de la personne
}

\author{
U. Thurnherr
}

1 Les médicaments antiviraux constituent, sous certaines conditions, une autre mesure de prévention, et donc une alternative au vaccin.

2 Cf. Müller DA. Campagne nationale de prévention de la grippe Nouveaux progrès en vue.

3 Panchaud C. Grippe et vaccination, un choix réfléchi. Soins infirmiers 2003;10:24.

Correspondance:

Pr Dr Urs Thurnherr

Pädagogische Hochschule

Postfach 111062

D-76060 Karlsruhe

E-mail:

urs.thurnherr@ph-karlsruhe.de
La principale raison invoquée en faveur de la vaccination du personnel médical est qu'il est, ou est amené à être, en contact avec des patients à risque pour lesquels une contamination pourrait entraîner de graves complications, voire la mort dans certains cas. On entend ici par personnel médical les membres du personnel en contact direct avec des patients lors de soins à domicile ou dans les hôpitaux, les cabinets médicaux ou les homes. Ce sont donc bien sûr les médecins et les soignants, mais aussi les physiothérapeutes, les ergothérapeutes, ou encore le personnel d'entretien, etc. Dans le contexte de la grippe, les patients sont dits «à risque» lorsqu'ils souffrent notamment de maladies cardio-vasculaires, de maladies des voies respiratoires ou d'immunodéficience. Ils peuvent être contaminés par le personnel, mais aussi par d'autres patients ou pensionnaires et, bien entendu, par les visiteurs. Pour prévenir les risques considérables liés à ces contacts, il faudrait que non seulement le patient lui-même soit vacciné, mais également toute personne le côtoyant. Le risque de contamination serait sensiblement plus faible si, en premier lieu, le personnel était vacciné [1].

\section{Prévenir les maladies, protéger l'autre, ne pas le mettre en danger}

Force est de constater qu'à ce jour, le personnel médical est peu enclin à se faire vacciner contre la grippe [2]. Pourtant, d'un point de vue médico-éthique, on pourrait se demander s'il n'est pas tenu moralement de le faire. En effet, au regard des principes évoqués dans ce contexte (tels que protéger au mieux ses patients et donc ne pas les mettre en danger) se faire vacciner semble revêtir un aspect moral. Bien des professionnels de la santé opposent pourtant à cette réflexion le fait que toute vaccination représentant une intervention sur le corps de la personne concernée, elle nécessite son libre consentement. Dans un article, Catherine Panchaud formule le dilemme en ces termes: «respect de la liberté individuelle et principe de ne pas nuire à l'autre» [3]. Une question se pose alors: Quelle décision prendre si je jouis de mon autonomie comme d'une liberté d'agir selon ma propre morale?

\section{Qu'est-ce que I'autonomie?}

Sur le plan éthico-philosophique, l'autonomie est synonyme de libre arbitre ou de liberté morale. Dans ce sens, être libre arbitre signifie décider seul ce que l'on doit faire compte tenu de ses convictions morales. A une question d'ordre moral je réponds selon ce que je crois être moral. Dans ce cas, ne pas se faire vacciner par indifférence ne peut donc pas être une réponse adéquate. Les questions morales nous aident à organiser le monde de manière à l'humaniser. Par conséquent, quand je prends des décisions de manière autonome concernant mes affaires, je cherche à définir selon quelles normes fondées, et donc raisonnables selon moi, nous devons vivre pour que le monde s'humanise. Je jouis de mon autonomie en me plaçant, compte tenu des conditions dans lesquelles je vis, de mes souhaits et de mes espoirs, des autres et de l'ensemble du monde extérieur, en position de m'organiser, le principe de cette organisation étant de se donner à soi même une loi d'humanité. L'autonomie, c'est-à-dire «ce qui se régit par sa propre loi», peut donc également se définir comme l'usage raisonnable de la liberté. A la lumière de ces réflexions, il me semble que si, en me faisant vacciner, je peux éviter à l'autre de tomber malade, voire de mourir des suites d'une grippe, je dois le faire au nom du principe de l'autonomie: en choisissant de me faire vacciner, je contribue à rendre le monde humain.

\section{Arguments contraires}

Si l'on observe l'argumentation contre la vaccination antigrippale du point de vue des professionnels de la santé, la question d'un éventuel devoir moral peut paraître un peu plus complexe. C'est pourquoi j'aimerais examiner ici de près quelques uns des arguments les plus souvent invoqués afin de déterminer s'ils ne rendent pas le principe de l'autonomie plus convaincant. Pour cela, nous devons consulter l'ensemble des informations pertinentes concernant la vaccination contre la grippe; l'autonomie est en effet toujours assortie de l'obligation de s'informer. 


\section{Autonomie et information}

La décision ne doit pas céder à l'humeur du moment mais s'appuyer sur toutes les informations disponibles. Pour que le principe de l'autonomie conserve sa valeur, il doit comprendre deux obligations: d'une part mettre des informations à disposition, d'autre part s'informer. Car seule une personne bien informée peut prendre une décision sensée. Les diverses campagnes de prévention de l'OFSP satisfont dans une large mesure à ce devoir d'informer les personnes de manière appropriée. Pour remplir cette obligation en respectant l'autonomie des destinataires, il convient de suivre certaines normes, nous n'en citerons que quelques unes: Tout d'abord, celui qui informe doit être honnête et présenter ses informations de manière objective. Il doit être compétent et fournir des informations aussi approfondies que possible. Celles-ci doivent, le cas échéant, comporter des indications quant à leurs sources et leur accessibilité. Elles doivent, en outre, devancer les questions des intéressés. Celui qui informe est tenu à tout moment de faire connaître ses propres intérêts. Seul celui qui remplit ces conditions peut transmettre à l'autre les connaissances nécessaires à une prise de décision libre, sans le manipuler, c'est-à-dire en respectant son autonomie.

\section{«Je ne suis jamais malade.»}

Autre argument souvent opposé: «Je n'ai pas besoin de me faire vacciner contre la grippe car je ne suis jamais malade, je prends beaucoup de vitamines $C$ et $j$ 'ai un excellent système immunitaire. Je n'infecterai donc personne.» En effet, quel serait l'intérêt de se faire vacciner contre une maladie que l'on ne risque pas d'attraper? Toutefois, cet argument comporte une inexactitude: en allemand, le terme de grippe désigne aussi bien un simple refroidissement entraînant des poussées de fièvre qu'une infection des voies respiratoires provoquée par les virus de l'influenza. Le terme allemand vient du mot français, lui-même originaire du russe «chripu», qui signifie enrouement [4]. L'enrouement étant un symptôme de l'une et l'autre des deux maladies, celles-ci ne sont pas différenciées au début. L'influenza, qui peut entraîner de sérieuses complications, se trouve donc considérée comme un refroidissement, sa gravité est donc largement minimisée. Si la vitamine C est certes susceptible de renforcer l'ensemble du système immunitaire, elle ne protège pas des virus de l'influenza. Et le fait que dans 20 à 30 pour cent des cas d'infec- tion par les virus de l'influenza la grippe ne se déclare pas ne constitue pas non plus un argument convaincant, car le virus peut être transmis par un porteur sain (c'est-à-dire une personne infectée ne présentant aucun symptôme de la maladie). De ce point de vue, le principe selon lequel il faut se faire vacciner contre la grippe pour protéger l'autre garde donc toute sa valeur.

\section{«Le vaccin rend malade.»}

Certains refusent de se faire vacciner par crainte que cela n'ait des effets sur leur santé, mentionnant le cas de personnes qui ont été malades à la suite d'une vaccination. Pourtant, les légers désagréments qui surviennent tout de suite après la vaccination dans environ 25 pour cent des cas et peuvent persister un ou deux jours sont sans comparaison avec les symptômes de la grippe. Quant aux effets secondaires graves, par exemple en cas d'allergie aux protéines aviaires, ils sont rarissimes. La tolérance au vaccin contre l'influenza, qui est de surcroît un «vaccin inactivé», s'est sensiblement améliorée ces dernières années. La nécessité de s'informer est donc ici particulièrement évidente. Il convient de respecter la décision de celui qui a de vraies raisons de croire que le vaccin peut nuire à sa santé; il ne s'agit pas de se faire du mal! Toutefois, ces craintes ne sont fondées que dans des cas très rares.

\section{«Le vaccin antigrippal est inutile.»}

Le pragmatique prétend que le vaccin est inutile puisque l'on peut attraper la grippe même si l'on est vacciné. Chaque année, un groupe d'experts s'appuie sur une analyse approfondie de la situation pour faire des pronostics concernant les souches du virus de la grippe attendues pour l'hiver à venir et déterminer ainsi la composition du nouveau vaccin. Ces pronostics se fondent sur les résultats de la surveillance mondiale de la grippe, qui observe continuellement les souches en circulation. Une optimisation du système de surveillance a permis d'accroître sensiblement l'efficacité du vaccin. Bien que les chances d'échapper à la grippe soient de 70 à 90 pour cent chez les jeunes et seulement de 50 à 70 pour cent chez les seniors, la vaccination antigrippale s'avère bénéfique même chez ceux qui attrapent la grippe après avoir été vaccinés: on a observé non seulement qu'elle freine l'évolution de la maladie, mais aussi qu'elle réduit le nombre de virus propagés en cas d'infection, limitant ainsi considérablement les risques de contamination,
Grimm J, Grimm W. Deutsches 1935. Col. 382-3. 
ce qui est primordial. Cet argument pessimiste et prétendument pragmatique ne peut donc pas être retenu.

\section{«Mon employeur cherche uniquement à limiter mon absentéisme.»}

Une partie une personnel soignant craint que l'on ne lui suggère de se faire vacciner uniquement dans le but de réduire l'absentéisme. Et nombreux sont les soignants qui estiment devoir refuser la vaccination pour se prémunir contre une telle instrumentalisation. Ce comportement, qui provient d'une certaine méfiance envers les institutions et le «système», atteint son apogée dans un discours exagérément polémique et un peu bizarre sur le droit de chacun de tomber malade [3]. En fait, l'expérience montre que ce groupe, du fait qu'il participe peu aux débats, a le sentiment que son autonomie n'est pas respectée. Les soignants travaillent dans des conditions d'autant plus délicates qu'ils sont souvent amenés à exécuter des décisions prises par d'autres. Pourtant, personne ne devrait accomplir une tâche à laquelle il est opposé ou pour laquelle il n'aura obtenu au moins un compromis. La problématique liée à l'autonomie du personnel soignant est encore méconnue et n'est pas suffisamment prise en considération dans le développement de l'organisation.

\section{Les campagnes contre la vaccination}

Des actions bien pensées visant à attirer l'attention, par un support du format d'un mouchoir en papier ou un dîner offert, conduisent parfois à des méprises. Car le personnel médical ne souhaite pas qu'on le séduise, il a besoin de convictions. Pour intéresser l'autre, il convient de s'adresser à lui en tant que personne autonome et de lui fournir des informations objectives sur un problème commun: si le personnel n'est pas disposé à se faire vacciner, l'institution ne peut pas protéger les patients à risque de manière optimale. Jouir de son autonomie signifie analyser le problème et le résoudre, ou contribuer à le résoudre, en accord avec ses propres convictions morales. Les responsables doivent envisager ce qui pourrait se produire au cours de la prochaine saison de la grippe. Par exemple, ils doivent imaginer des scénarios où il faudrait gérer une pandémie et, en même temps, traiter un certain nombre de cas de SRAS. Dans une telle situation, un fort taux d'absentéisme aurait des conséquences dramatiques. Il convient donc d'arriver ensemble à des solutions parmi lesquelles se trouve la décision du personnel de se faire vacciner chaque année contre la grippe, une décision prise librement et en toute connaissance de cause. On ne peut toutefois espérer la participation et l'engagement de l'autre que si son autonomie a toujours été respectée, et non pas uniquement lorsque l'on en a besoin. En outre, on peut se demander s'il ne serait pas préférable que les campagnes contre la grippe soient initiées et menées par une autre institution que l'employeur.

\section{En bref}

J'arriverai peut-être à la conclusion que la vaccination n'est pas judicieuse dans mon cas, par exemple en raison d'une allergie, et, de ce fait, j'estimerai ne pas être tenu de me faire vacciner. Dans certaines circonstances, d'autres motifs de refus peuvent également se justifier sur le plan moral, et une décision motivée par des convictions morales doit toujours être respectée. Dès lors, il ne peut être question d'un devoir moral absolu de se faire vacciner contre la grippe. Néanmoins, celui qui revendique son droit à l'autonomie doit traiter la question concernant la protection de l'autre d'un point de vue moral. En d'autres termes, s'il n'y a pas de devoir moral de se faire vacciner, il y en a un à réfléchir au problème et à contribuer à le résoudre. En ce qui concerne la vaccination antigrippale, je suis convaincu que l'usage de la liberté morale et la jouissance de l'autonomie devraient amener les membres du personnel, dans leur grande majorité, à se faire vacciner de leur propre gré. 